\title{
Silica coated nanoparticles: synthesis, magnetic properties and spin structure
}

\author{
F. Mazaleyrat \\ SATIE, ENS Cachan, CNRS, UniverSud, 61 av President Wilson, F-94230 \\ CACHAN, France. \\ M. Ammar, M. LoBue \\ SATIE, ENS Cachan, CNRS, UniverSud, 61 av President Wilson, F-94230 \\ CACHAN, France. \\ J-P. Bonnet, P. Audebert \\ PPSM, ENS Cachan, CNRS, UniverSud, 61 av President Wilson, F-94230 \\ CACHAN, France. \\ Y. Champion \\ ICMPE, CNRS, 15 rue Georges Urbain, F-94070 Vitry-sur-Seine, France \\ M. Hÿtch, E. Snoeck \\ CEMES, CNRS, rue Jeanne Marvig, F-31055 Toulouse, France
}

\begin{abstract}
In the recent years magnetic nanoparticles have been extensively studied for their superparamagnetic properties providing useful labels in biology or for fundamental aspects including the size dependence of magnetic atomic moment and the effect of surface anisotropy. In most cases, the particles were smaller than $10 \mathrm{~nm}$ and interestingly, the sizes ranging between 10 and $100 \mathrm{~nm}$ have been poorly investigated until now. This is mainly due to the fact that usual chemical routes produce 5$10 \mathrm{~nm}$ oxide or metallic particles or eventually $20 \mathrm{~nm}$ at most. On the over side, atomization techniques yields particles in the micrometer range. Metallic particles are particularly interesting for better magnetic propeties compared to oxides, but they have two big drawbacks: they are not biocompatible and they are conducting electricity. Consequently, it's necessary to produce core-shell particles, for witch the shell is biocompatible and insulating and with a perfect control of thickness and uniformity of that shell. In this work, we study metallic particles synthetized by an original evaporation-condensation techniques that produces particles of sevral tens
\end{abstract}


of nanometers. We prepared hard magnetic cobalt particles and soft FeNi ones by the cryogenic metlting process coated by a silica shell using sol-gel metod. Morphological and magnetic properties are presented, showing the efficiency of ultrasonic sol-gel process for that purpose.

Key words: Core-shell particles, nanomagnetism, ultrasonic synthesis PACS: $81.20 . \mathrm{Fw}, 7550 . \mathrm{Tt}, 7525 .+\mathrm{z}$

\section{Introduction}

Magnetic nanoparticles have attracted increasing interest in the recent years. The reasons are actually fundental as well as applied: many issues on fundmental magnetism of nanoparticles are still unsolved and experiments at the scale of individual particle is lacking. For exemple, although theory is quite well established, systematic experiments on the criticle size at which the magnetic structure changes from single-domain to vortex or multi-domain is still missing . From practical point of view, magnetic nanoparticles have a great potential in biomedical applications and high frequency electronics.

Superparamatic particles (SPMP) are widly used in in biology. Indeed, their are many application of superparamagnetic beads as image contrast agent in magnetic resonance imaging and hyperthermia therapy but essentially for magnetophoresis (drug carriers, magnetic concentration...). For all applications, superparamagnetic behavior is necessary because a zero net magnetization under zero magnetic field prevents agglomeration of magnetic beads. For magnetophoresis, because relatively high DC magnetic field can be produced by permanent magnet systems, essentially the volume of magnetic particles is important, so classical beads composed of $50 \%$ of $5 \mathrm{~nm}$ in diameter magnetite and/or maghemite particles are perfectly fitted. Moving beads by application of a gradient field is one thing but it would be even more interesting to locate and/or quantify this beads. Various principles for the detection of magnetic beads have been proposed $(1 ; 2 ; 3 ; 4)$ but all these systems are limted in sensitivity by the magnetic properties of the particles. As it was demontrated by Néel (5), the suceptibilty of a SPMP at a given temperature is essentially dependent on its volume and saturation magnetization:

$$
\chi=\frac{J_{S}^{2} V}{3 \mu_{0} k_{B} T}=\alpha J_{S}^{2} D^{3} .
$$

This formula is valid if two conditions are satisfied:

Email address: mazaleyrat@satie.ens-cachan.fr (F. Mazaleyrat).

URL: http://www.satie.ens-cachan.fr (F. Mazaleyrat). 
- the volume of the SPMP has to be small enough for the magnetization to be relaxed, i.e. if the thermal energy of the particle exeeds the anisotropy enregy

$$
K_{1} \frac{\pi D_{S P}^{3}}{6}=\ln \frac{f_{0}}{f} k_{B} T
$$

- the particle has to be single domain (SD), i.e. its diameter is smaller than the critical one, $D_{S D}$, where vortex and domain formation are expected to be energetically favourable; $D_{S D}$ can be estimated e.g. following Bertotti (6) for a soft particle

$$
D_{S D} \approx 4 \sqrt{3} \ell_{e x}
$$

where $\ell_{e x}=\sqrt{2 \mu_{0} A / J_{S}^{2}}$. Indeed, considering the diversity of soft magnetic materials and many factors affecting their behavior, finding the ideal SPMP is not trivial. On Fig. 1, the values of $D_{S P}$ and $D_{S D}$ for most common soft magnetic mateirals have been reported as a function of saturation magnetization together with iso-susceptibility curves plotted using equation (1). The best materials are that having their lower critical diameters on the highest iso-susceptibility curve. It is clear that metallic materials leads to the best compromise between size, magnetization and anisotropy. From magnetic point of view, FeNi alloys are particularly suitable for that purpose in particular due to their low anistropy. In contrast, because of the toxicity of $\mathrm{Ni}$, the particles have to be coated by a stable biocompatible layer.

Concerning high frequency electronics, nanoparticles have potential especially in power applications. It is well known that metallic thin films exhibits high permeability at high frequency, but applications are limited to low power applications because the volume of the material is necessarilly very small. In contrast, nanoparticles can be used for realization of bulk components. The frequency limits of magnetic particles are mainly due to three phenomena:

- gyroresonance which is controlled by magnetization and anisotropy only;

- eddy current relaxation wich can be controlled using particles smaller than the skin depth;

- domain wall resonance which can by suppressed by using single domain particles.

Again metallic particles are interesting because of their high magnetization and the possibility to change the anisotropy constant. If large permeabilty is required, ultra soft materials such as permalloy can be used. On the contrary, if high frequency is needed, higher anisotropy materials such as cobalt can be chosen but in all case, insulation will be necessary. However, insulation with a non magnetic layer affects the permeability of the material. If the relative volume of non magnetic coating is small, Non Magnetic Grain Boundary models 


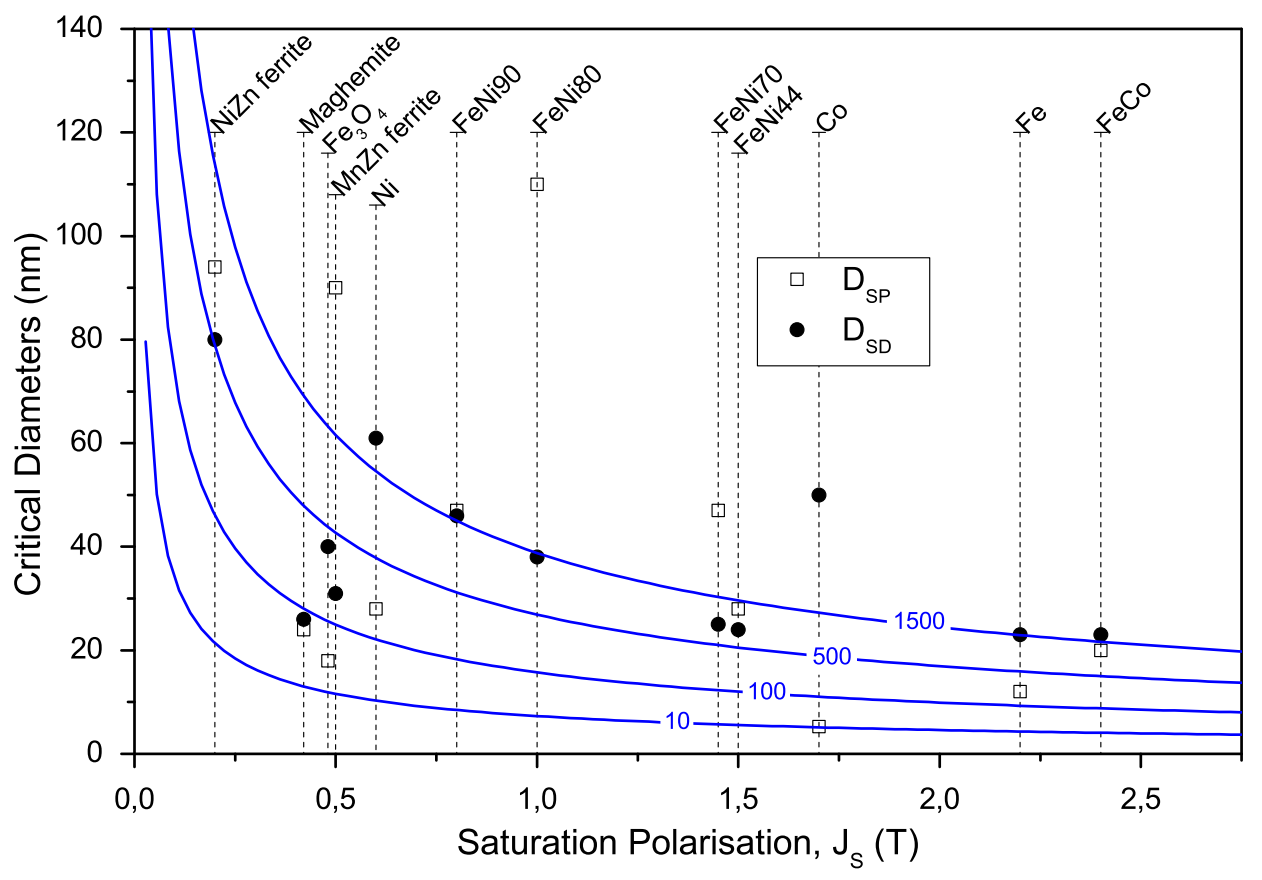

Figure 1. Critical particle size for different soft magnetic materials. Open squares, superparamagnetic limit; colsed circles single domain limit; continuous lines, iso-susceptibility curves computed from eq. 1 (labels are for the values of $\chi$ ).

applies:

$$
\chi_{e f f}=\frac{\chi_{i}}{1+\chi_{i} \frac{t}{D}}
$$

where $g$ is the average gap between particles, i.e. approximatively twice the thickness coating. This equation clearly demonstrate that the coating has to be much smaller than the particle size. Considering that particles are smaller than $100 \mathrm{~nm}$, it is clear that coating must embed each particle individually with perfect homogeneity and nanometer controll of its thickness.

For these purposes, our approch is to start from metallic particles produced by cryogenic melting (derived from classical evaporation-condensation method) and to grow an inorganic dielectric layer by sol-gel technique. In order to reach the objective in terms of control of homogeneity and thickness of the dielectric layer, a improvement of Stöber method by use of high power density ultrasounds has been developped. 


\section{Experimental details}

Synthesis of nanoparticles. Co and FeNi particles have been produced by cryogenic melting, a method consisting in overmelting a droplet of metal by induction kipping it in levitation in the cryogenic media (usually $\mathrm{LN}_{2}$ ). The vapor condensate in the calefaction layer around the droplet and further coalescence yields solidification into $20-100 \mathrm{~nm}$ particles, the average size depending on the metal, the cryogenic medium and the temperature of the melt. Particles are then collected in hexane where a passivation layer of 2-4 $\mathrm{nm}$ forms at the surface of the particles. Cobalt have been chosen for its high anisotropy favorable to high frequency applications. Co particles are fcc, have an average size of $52 \mathrm{~nm}$ (standart deviation of $23 \mathrm{~nm}$ ) and a surface layer composed of $\mathrm{CoO}$ and hydroxides. Permalloy was chosen for its low anisotropy favorable to high permeability and SPM properties. Concerning FeNi, because metals have different vapor tension, the composition of vapor (and so that of particles) may differ from that of melt. To avoid the composition drift during the process, we have choosen the homoazeotropic composition $\mathrm{Fe}_{29.5} \mathrm{Ni}_{70.5}$ (7). The average grain size is $50 \mathrm{~nm}$ (20 nm standart deviation) and the surface layer is composed mainly of $\mathrm{NiO}$ and nickel hydroxides.

synthesis of silica coating. The silica shell onto particles was synthesized according to the Stöber method [23] (sol-gel reaction). Firstly, cobalt particles (typically $80 \mathrm{mg}$ ) have been dispersed in cycloexane, a polar solvant miscible with APTES (Amino-Propyl-Triethoxysilane) and oleic acid (which plays the role of surfactant) during 90 minutes under a controlled ultrasonic excitation of $3 \mathrm{~W} . \mathrm{cm}^{-3}$ powered by a $200 \mathrm{~W}$ horn. The surfactant is necessary to disperse cobalt particles due to the strong magnetic forces. Then, APTES and oleic acid are introduced in order to functionalize the surface with amine groups $\left(\mathrm{NH}_{2}\right)$ which present a good affinity with metal and to start the polymerization with the silica precursor fixed on the end of the APTES chains. In a third step, different quantities of tetraethylorthosilicate (TEOS) and ammonia were successively introduced into the suspensions to grow the silica layer. Sonication $\left(0.5 \mathrm{~W} . \mathrm{cm}^{-3}\right)$ was maintained during the sol-gel reaction (90mn). Finally the suspensions are centrifuged at $3000 \mathrm{rpm}$ for 10 minutes, the solvent is discarded, the nanoparticles are again ultrasonically dispersed in ethanol and dryed for sample preparation. In the case of FeNi particles, the functionalization step was not necessary. Indeed, nickel hydroxides have an isoelectric point at $\mathrm{pH}=12$, whereas the mixture has a $\mathrm{pH}=8$. Thus, the surface of particles is positively charged and have a good affinity with negatively charged silica. Consequently, dispersion was conducted simply in ethanol and the polymerisation starts in the secod step with hydrolization of TEOS.

Characterization. The magnetic properties have been measure between room and $\mathrm{LN}_{2}$ temperature using standart VSM. Morphology and composi- 
tion of particles were analysed using a TECNAI F20 HRTEM. Additionally, Electron Energy Loss Spectra (EELS) were recorded using a Gatan Image Filter attachement. Holography experiments were performed on a Spherical Aberration Corrected TEM Tecnai F20 (FEI) fitted with an objective lens aberration corrector (CEOS) and rotatable electron biprism. The field emission gun (FEG) provides the highly coherent electron source necessary for electron holography. Holograms were recorded with a biprism voltage of $90 \mathrm{~V}$ on a Gatan $1 \mathrm{k} \times 1 \mathrm{k}$ slow scan CCD camera and processed using a dedicated software, developed in the Gatan Digital Micrograph environment. Exposure times were typically of the order of $4 \mathrm{~s}$. Objective lens was used to magnetise the sample in two opposite directions by tilting the sample holder. The two holograms recorded at remanence were substracted to eliminate electrostatic potential contribution to the phase shift of electrons passing through the sample. The phase shitf $\phi_{M a g}$ is sensitive to the in-plane (perpandicular to electron beam) component of the induction, according to $(8 ; 9)$ :

$$
\phi_{M a g}=-\frac{e}{\hbar} \int A(x, y) d z=-\frac{e}{\hbar} \iint B_{\perp}(x, y) \mathrm{d} x \mathrm{~d} z
$$

\section{Results and discussion}

After coating, particles have been dryed and observed by TEM. Picture (a) in Fig. 2 shows and exemples of Co particles coated a $8 \mathrm{~nm}$ layer of silica. The layer is not perfectly regular but globally uniform and each particle appears separatly coated. By increasing the TEOS concentration, the coating can be as thick as $80 \mathrm{~nm}$, particles are still separated and the layer is uniform (b). This result is attributed to the the effect of high power density ultrasounds which avoid particle agglomeration and favours uniformity of the sol-gel reaction on the particles. Same results are found qualitativlely with FeNi particle without surface functionalisation as it is shown on picture (c) and (e). Additionally, Electron Energy Loss Scattering has confirm that silicon is exclusively located at the surface of the particles and HRTEM gives evidence of the homogeneity and of the amorphous nature of silica coat. The main difference compared to the technique using an APTES functionalization step, is that the efficiency is reduced at high TEOS concentration.

Fig.3 shows the hysteresis loops of coated Co nanoparticles. The specific magnetization decrease consistently with increasing the volume of TEOS. By comparing the saturation values with respect to raw particles and considering spherical particles of averadge diameter $D$ surrounded by a shell of thickness $t$, it was possible to extract $t$ from the data (see Table 1 ). This values are in good agrement with TEM measurements which confirms that TEM samples are representative of the whole. As expected, Co particles are expected, particles are strongly hysteretic because all particles are larger than the critical superparamagnetic size $\left(D_{S P}=7.5 \mathrm{~nm}\right)$ and mainly single domain 

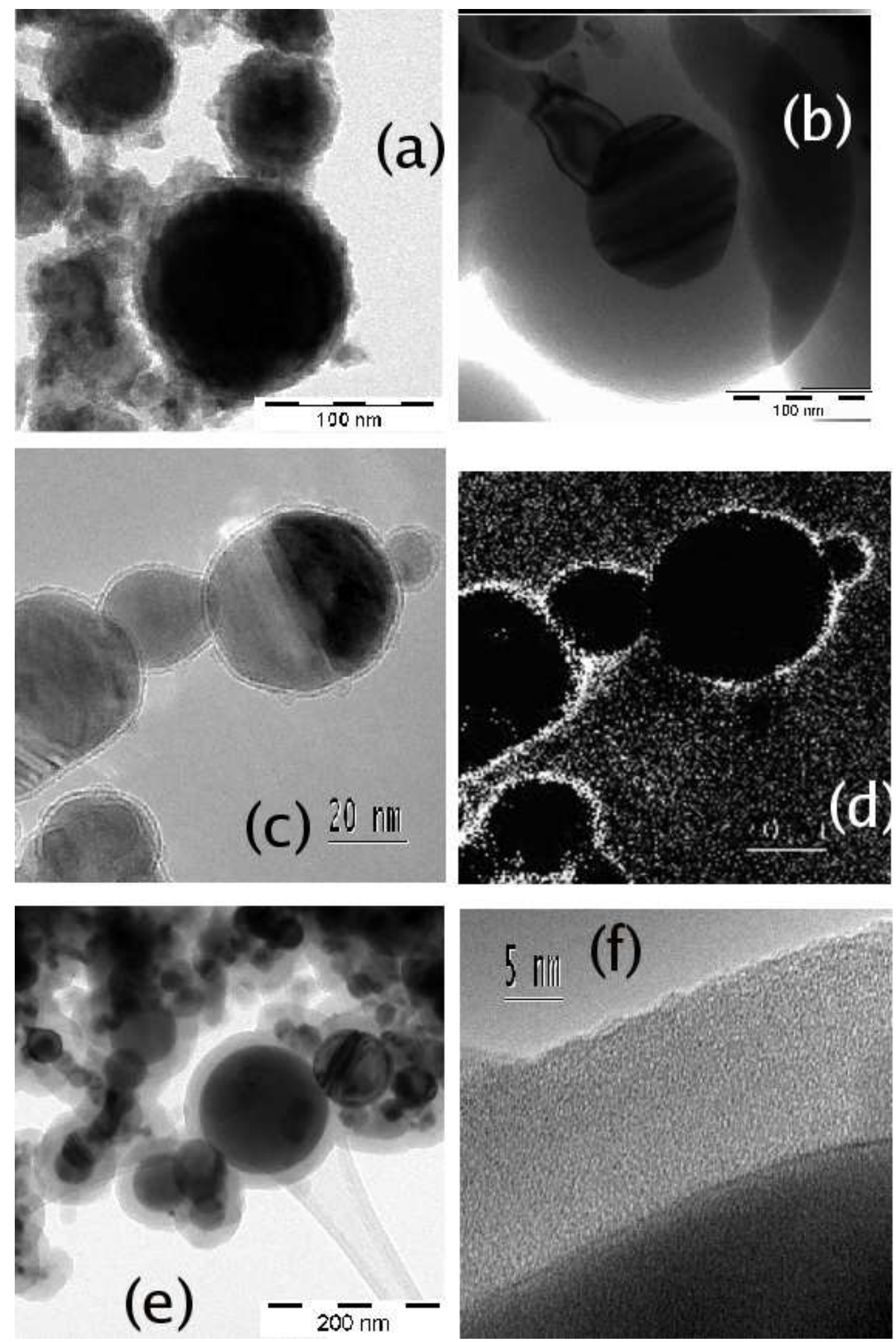

Figure 2. TEM pictures of core-shell nanoparticles. Cobalt particles with a thin (a) and thick (b) layer of silica. FeNi particles with a thin layer of silica: (c), conventional image, (d) silicon map by EELS. Thicker layer on FeNi particles, conventional TEM (e); HRTEM (f). 


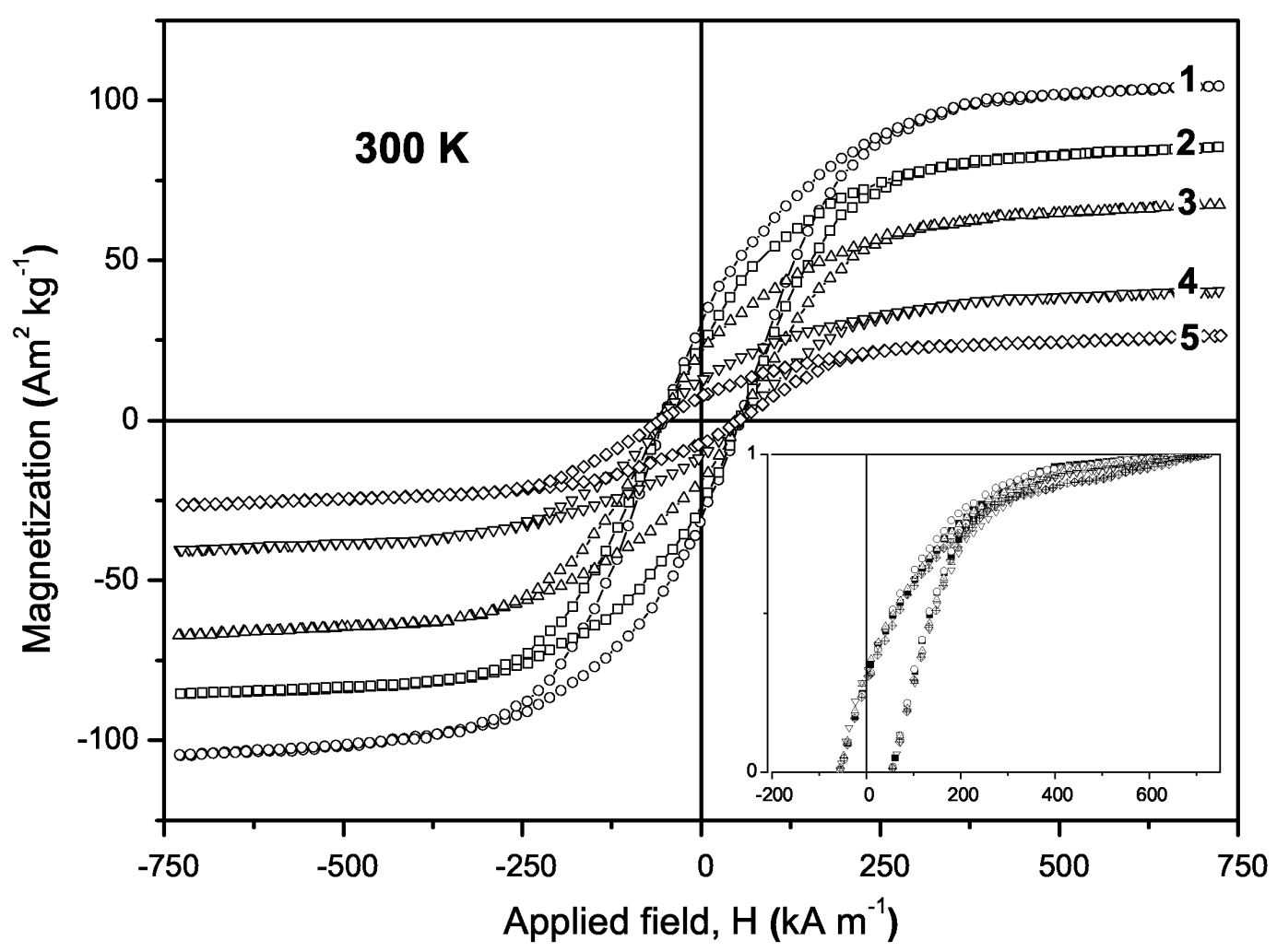

Figure 3. Hysteresis loops of $\mathrm{Co} @ \mathrm{SiO}_{2}$ nanoparticles. Inset: the same curves plotted with respect to reduced magnetization $M / M_{S}$.

Table 1

Thickness dependence of silica shell on TEOS concentration measured by TEM and deduced from specific saturation magnetization

\begin{tabular}{|c|c|c|c|c|c|}
\hline \multicolumn{3}{|c|}{$\mathrm{Co}$} & \multicolumn{3}{|c|}{$\mathrm{Fe}_{30} \mathrm{Ni}_{70}$} \\
\hline $\begin{array}{c}\text { TEOS } \\
(\mu \ell)\end{array}$ & $\begin{array}{l}t_{T E M} \\
(\mathrm{~nm})\end{array}$ & $\begin{array}{c}t_{M A G} \\
(\mathrm{~nm})\end{array}$ & $\begin{array}{c}\text { TEOS } \\
(\mu \ell)\end{array}$ & $\begin{array}{l}t_{T E M} \\
(\mathrm{~nm})\end{array}$ & $\begin{array}{l}t_{M A G} \\
(\mathrm{~nm})\end{array}$ \\
\hline 100 & 8 & 7 & 50 & 3 & 4 \\
\hline 200 & 13 & 12 & 100 & 8 & 9 \\
\hline 300 & 44 & 43 & 200 & 15 & 17 \\
\hline 400 & 80 & 71 & 500 & 33 & 24 \\
\hline
\end{tabular}

$\left(D_{S D} \approx 50 \mathrm{~nm}\right.$, see Fig.1). In the inset of Fig.3, loops are plotted with respect to reduced magnetization showing clearly that corcivity $(55 \mathrm{kA} / \mathrm{m})$ is independent of silica shell thickness. This indicates that the main source of anisotropy in this system is magnetocrystalline. The theoretical coercivity for an enssemble of randomly oriented particles is given for cubic particles, after Néel (10), by $H_{C}=0.64 K_{1} / J_{S}$. This yields the value of $146 \mathrm{~kJ} . \mathrm{m}^{-3}$ for the anisotropy constant of fcc-Co.

Concerning FeNi particles, consistent values of thickness are deduced from 
magnetic mesurements as previously (see Table 1). However, concerning hysteresis, the observed behavior is mutch different compare to the case of Co. First we remark that the coercive field is quite strong in spite of the fact that most of the particles have a size below the superparamagnetic limit (71 $\mathrm{nm})$ is larger than most of particles. Secondly, $H_{C}$ is much larger than expected by applying above mentionned Néel formula $(350 \mathrm{~A} / \mathrm{m})$. These two phenomenons can be explained by shape anisotropy that can be relevent for even for nearly sperical particles because of the nearly zero magnetocrystalline anisotropy. Following Néel (11), the demagnetizing factor of a slightly elongated spheroid, having a ratio between lenghts of small and long axes $\gamma$, can be written $N_{\|}=\frac{1}{3}\left(\frac{9}{5}-\frac{4}{5} \gamma\right)$. Knowing that $N_{\perp}+2 N_{\|}=1$, it's easy to show that the shape anisotropy is $K_{S}=-\frac{\gamma-1}{5 \mu_{0}} J_{S}^{2}$. Consequently, the observed coercivity can be explained using Néel-Stoner result for uniaxial anisotropy (12):

$$
H_{C}=0.96 \frac{K_{S}}{J_{S}}=0.192 \frac{(\gamma-1) J_{S}}{\mu_{0}}
$$

if $\gamma-1 \approx 20 \%$. Refeering to TEM pictures, this difference in length between long and short axis is actually unrealistic, showing that the observed coercivity has more complex origin. It is also interesting to note that the coercivity varies sensitively with thickness coating. The decrease observed for thiner silica layer can be due to a diminution of dipole-dipole interactions as distance between particles increase. On the other hand, the increase observed for thicker layers is crearly related with stress applied by the coating as it has been shown by Fourrier Transform Infra red spectroscopy (13).

According to previous remarks, the addition of a shape anisotropy term change the particle energy balance. As a consequence their spin structure can be deeply modified (until now they were supposed single domain). To investigate the spin structure, electron holography experiments have been conducted. TEM samples have been prepared by dispersing particles by ultrasound in ethanol and a carbon grid was dipped in the suspention. This dispersion is necessary to avoid long chain formation that influences deeply the magnetic structure (14). Due to the magnetic interactions, it was not possible to observe individual particles. However, it was possible to isolate very small groups of 2-3 particles and to record holograms. Pictures of the particles and the corresponding greyscale phase plot are shown in Fig.5. In this plot, the in-plane component of induction - and thus magnetization - is tangential to the isophase lines showing a vortex configuration where spins curls in the plane. In the center of the biggest particle, the white color indicates a maximum in the phase revealing that in-plane induction is going to zero which means that spins turns out of plane. To go more insight the magnetic structure, phase profile along a line going through the biggest particles have been plotted as a function of radius (the originis the middle of central particle) according to the line drawn on the picture. The in-plane component of induction $\left(B_{\theta}\right)$ is 


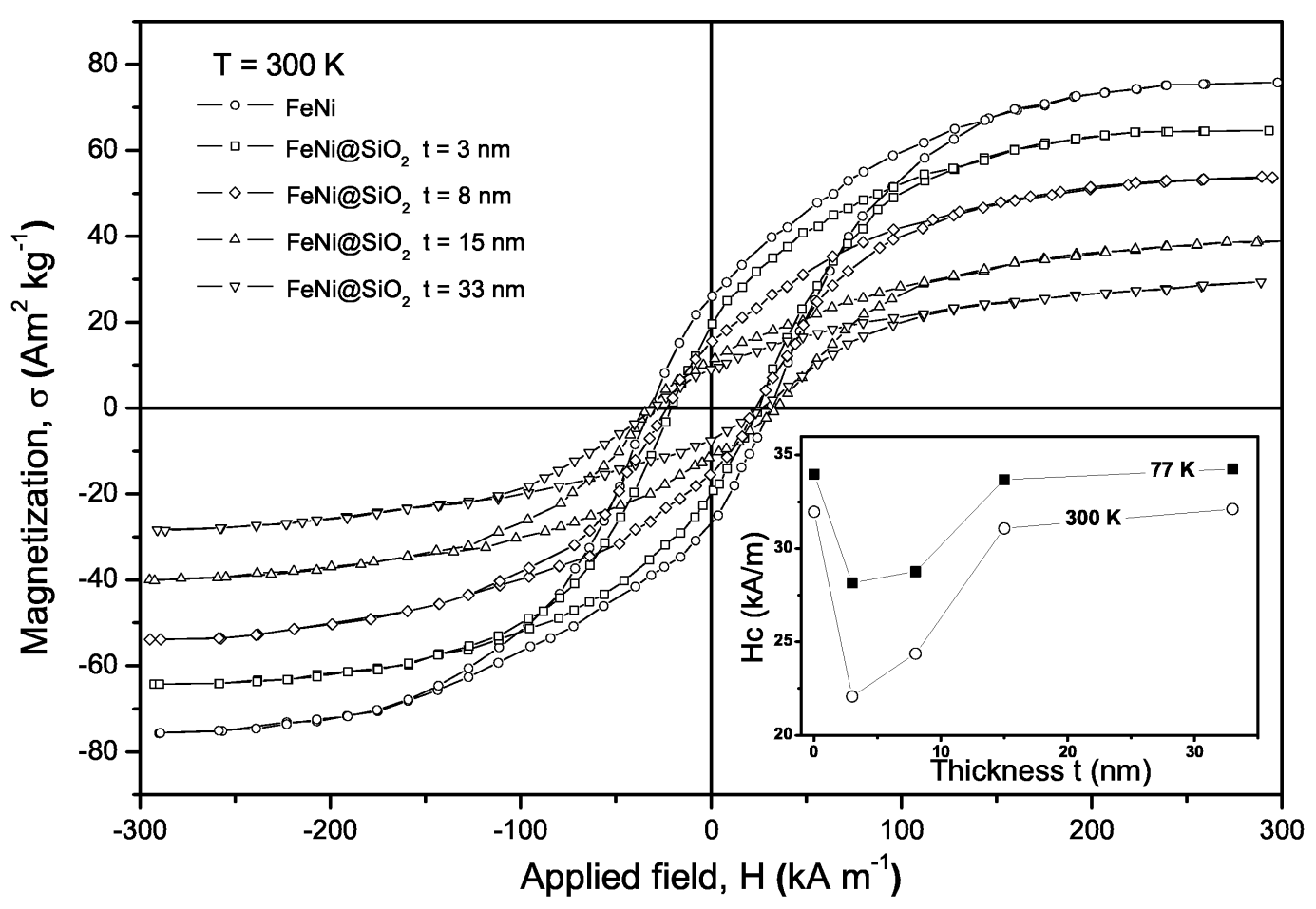

Figure 4. Hysteresis loops of $\mathrm{Fe}_{29.5} \mathrm{Ni}_{70.5} @ \mathrm{SiO}_{2}$ nanoparticles

deduced by derivation of the phase taking into account the depth variation. If the particle is considered perfectly spherical equ.5 yields:

$$
B_{\theta}(\rho)=-\frac{\hbar}{e} \times \frac{1}{z(\rho)} \times \frac{\mathrm{d} \phi_{M a g}(\rho)}{\mathrm{d} \rho} .
$$

The derivation of phase profile was possible due to the exeptional stability of the microscope and exellent signal to noise ratio of the acquisition system. Fig.6 depicts the phase shift deduced from holograms and the calculated in duction. The shape of induction distribution across the diameter is consistent with a vortex having an out-of-plane core $\left(B_{\theta}=0\right.$ in the center). Out of the central core, $B_{\theta}$ rapidly increases and reach the maximum value of $0.87 \mathrm{~T}$, close to that expected by magnetic measurements (the saturation polarisation of the core is estimated to $1.0 \mathrm{~T}$ ). In the oxide shell, $B_{\theta}$ goes again to zero rapidly because the shell is not magnetic. As predicted, the vortex configuration is clearly attested here with well defined core and curling zones. Indeed, it is possible to calculate the sigle domaine critical size from eq. 3. Taking $\mathrm{Fe}_{30} \mathrm{Ni}_{70}$ values for the exchange constant $A$ and the saturation magnetization $J_{S}\left(10 \mathrm{pJm}^{-1}, 1 \mathrm{~T}\right.$ and $700 \mathrm{Jm}^{-3}$, respectively) yields $\ell_{e x}=\sqrt{2 \mu_{0} A / J_{S}^{2}}=5 \mathrm{~nm}$, and we find that the particle $(40 \mathrm{~nm})$ is sensitively larger than $D_{S D} \approx 35 \mathrm{~nm}$.

This experimental distribution of magnetisation in the vortex have been compared to Usov model (15). This model is based on on Brown micromagnetic approach (16) considering a cylindrical particle in the limit of vanishing anisotropy in which the magnetisazation distribution is divided in two re- 


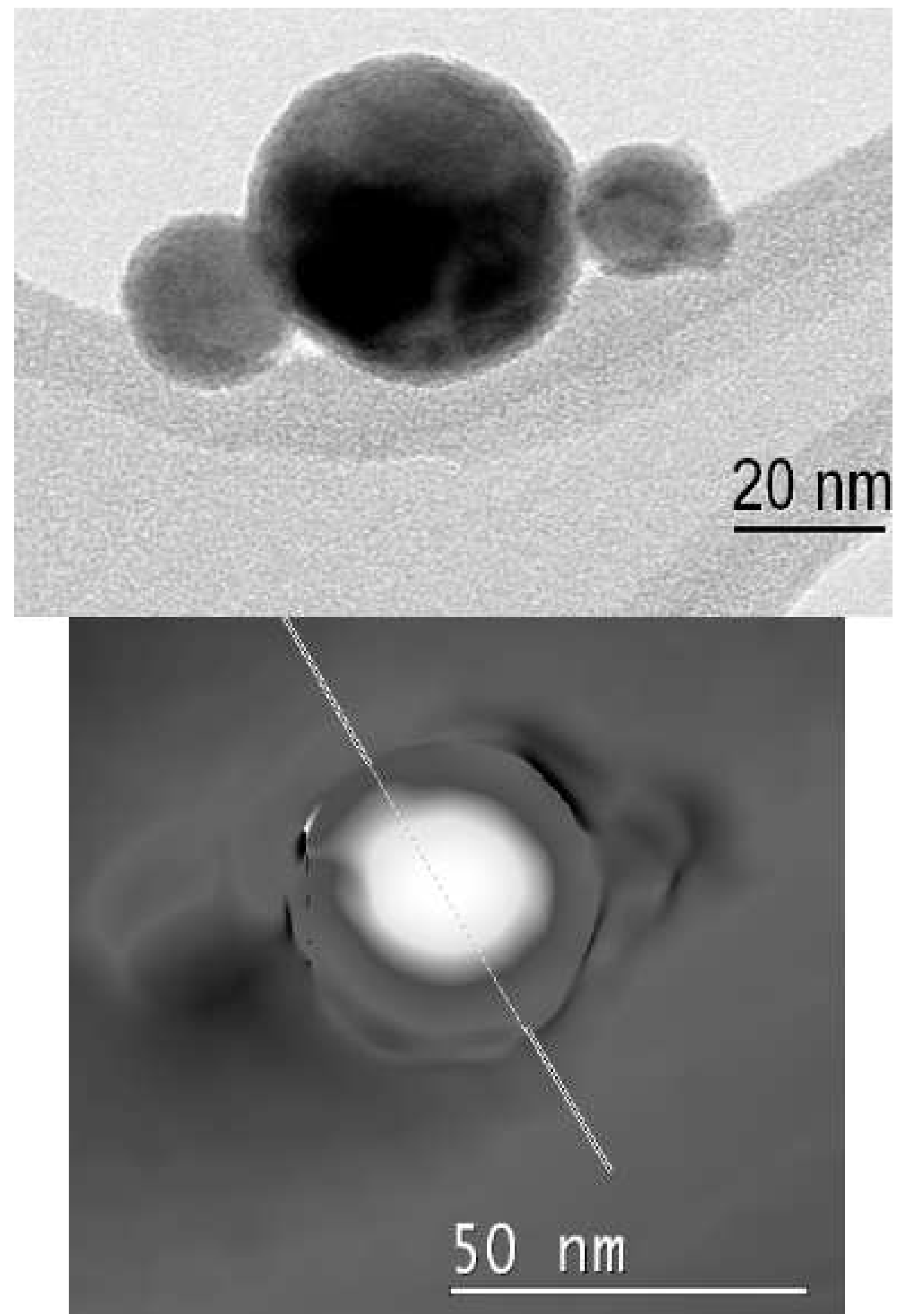

Figure 5. Tom: TEM picture of the studied group of particles. The central particle has a diameter of $40 \mathrm{~nm}$. Bottom: computed iso-phase map. White is for maximum phase shift corresponding here to out of plane magnetization, back is for zero. The line shows the path along which the induction is computed. 


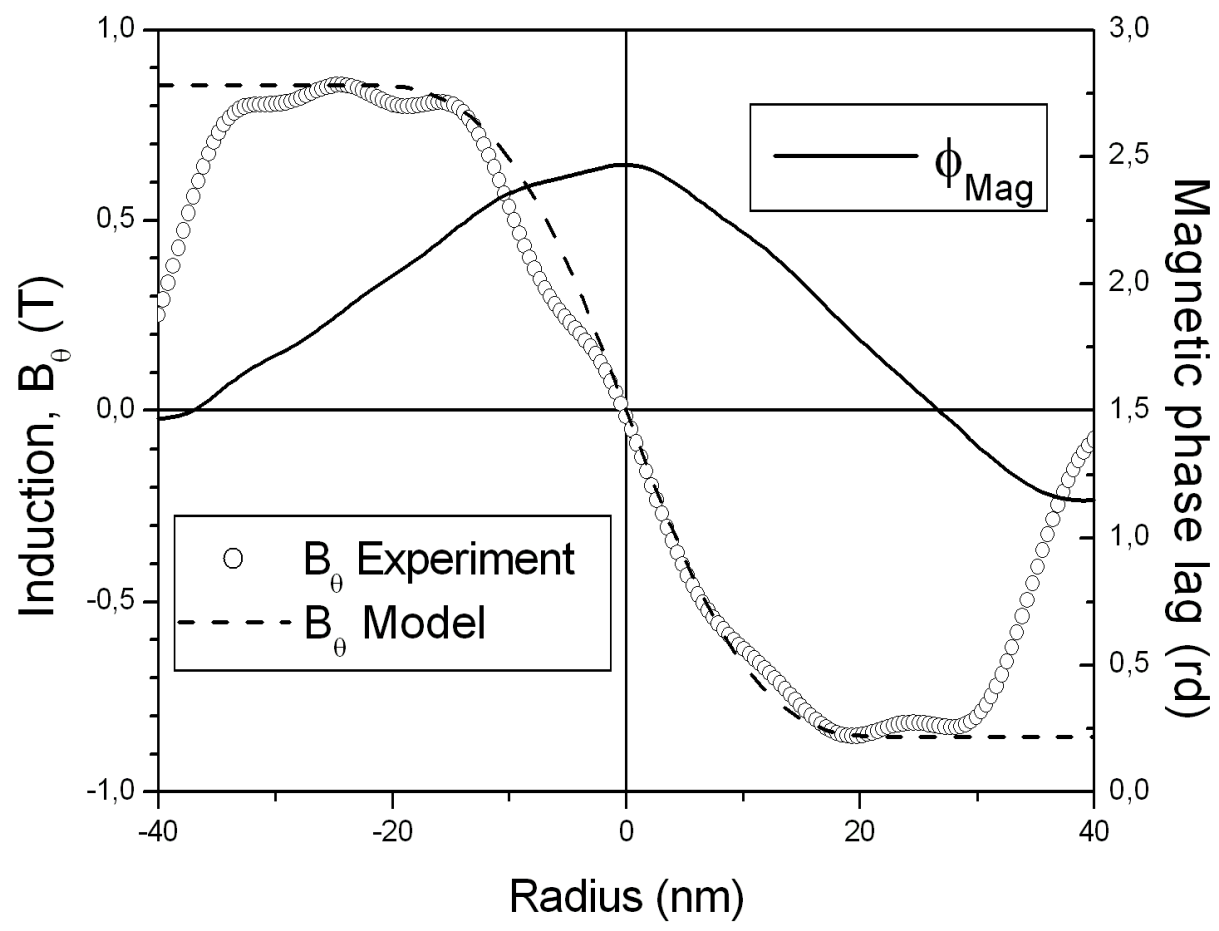

Figure 6. Magnetic phase shift mesured along the line shown in fig. 5. In-plane magnetization (here equatorial component $B_{\theta}$ ) compared with Usov model. On the border of particle, magnetisation goes to zero due to post-precessing (the thickness becomes zero).

gions: an outer region where the magnetisation curls in-plane and a core region where the magnetisation turns gradually out of plane. Under these conditions, Brown's equation can be solved analytically and the magnetisation distribution is given by:

$$
\left\{\begin{array}{l}
m_{\theta}=\frac{2 a r}{a^{2}+r^{2}} \text { if } \quad 0 \leqslant r \leqslant a \\
m_{\theta}=1 \text { if } a<r \leqslant D / 2
\end{array}\right.
$$

where $r$ is the radius of the particle and $a$ the radius of the vortex core. By fitting experimental data with model, one can obtain the effective exchange length given by $\pi \ell_{e x}^{*}=2 a$, so that $\ell_{e x}^{*} \approx 8 \mathrm{~nm}$. This effective exchange length has to be compared with the intrinsic exchange length $\ell_{e x}=5 \mathrm{~nm}$ and the domain wall parameter $\ell_{w}=\sqrt{A / K_{1}}=120 \mathrm{~nm}$ (the magnetocrystalline anisotropy of $\mathrm{Fe}_{30} \mathrm{Ni}_{70}$ is $700 \mathrm{Jm}^{-3}$ ). This experiment shows that the vortex configuration is not ruled by classical exchange lengths but by an effective one that have an intermediate value, presumably dependent of the size of the particle itself. The magnetic processes involved are different from that of coherent rotation and domain wall displacement. Previous micromagnetic simulation 
have indicated that the coercivity is linked to the critical fields at which the vortex nucleates or flips (17) and since these fields are intimatly dependent to $\ell_{e x}^{*}$ it can be inferred that the coercivity is strongly size dependent in this configuration.

\section{Conclusion}

Metallic core-shell particles have been sucessfuly produced by silica coating of $50 \mathrm{~nm}$ Co and FeNi particles. Sol-gel synthesis under high power ultrasounds allowed to coat each particle individually with a nm-controlled uniform silica layer. Magnetic mesurement have shown that cobalt particles behaves globally like single domain particles as it was expected. In contrast, the FeNi particles do not exhibit superparamagnetism because the shape anisotropy dominates the magnetocrystalline anisotropy. In addition, particles are magnetically harder than expected even taking the shape anisotropy in consideration. Since electron holography has confirmed the formation of spin vortex in the particles in this size range, it is supposed that the high coercivity of these extremly soft nanoparticles is due to the peculiar magnetic processes in that magnetic configuration. Summerizing, we have demonstrated that nanoscale control of silica coating on metallic particle si possible with very good homogeneity and reproducibility. Co particles hexibited the expected behavior and are good candidate for high frequency applications. In contrast, FeNi particles, in spite of the biocompatible coating, cannot be used when superparamagnetic behaviour is required. For this purpose, it will be necessary to produce particles at least under $40 \mathrm{~nm}$ and with reduced size dispersion.

\section{References}

[1] M. B. Simmonds, US Patent 6,046,585 (1997).

[2] P. I. Nikitin, P. M. Vetoshko, US Patent 20030027197A1 (2003).

[3] US Patent 6,518,747.

[4] US Patent 6,743,639.

[5] L. Néel, Influence des fluctuations thermiques sur l'aimantation des grains ferromagnétiques très fins, Comptes Rendus.Acad.Sci. 228 (1949) 664666.

[6] G. Bertotti, Hysteresi in magnetism, Academic Press, San Diego, 1998.

[7] Y. Champion, J.-L. Bonnentien, C. Langlois, C. Duhamel, J. Moulin, F. Mazaleyrat, P. Bayle-Guillemaud, M. J. Hytch, Synthesis and processing of metallic nano-powders for the study of their mechanical and magnetic properties., Mat. Sci. Forum 426-432 (2003) 2411-2416.

[8] A. Tonamura, T. Matsuda, J. Endo, T. Arii, K. Mihama, Direct observation of fine structure of magnetic domain walls by electron holography, Phys. Rev. Lett. . 
[9] R. E. Dunin-Borkowski, M. R. McCartney, D. J. Smith, S. S. Parkin, Ultramicroscopy 74 (1998) 61-67.

[10] L. Néel, Le champ coercitif d'une poudre ferromagnétique cubique à grains anisotropes, Comptes Rendus.Acad.Sci. Paris 224 (1947) 1550-51.

[11] L. Néel, Propriétés d'un ferromagnétique cubique en grains fins, Comptes Rendus. Acad. Sci. 224 (1947) 94-96.

[12] E. Stoner, E. Wohlfarth, Mechanism of magnetic hysteresis in heterogeneous alloys, Trans. Roy. Soc. A 240 (1948) 599-644.

[13] M. Ammar, F. Mazaleyrat, J. Bonnet, P. Audebert, A. Brosseau, G. Wang, Y. Champion, Synthesis and characterization of core shell structure silica coated $\mathrm{Fe}_{29.5} \mathrm{Ni}_{70.5}$ nanoparticles, Nanotechnology 18 (2007) 285606.

[14] M. J. Hÿtch, R. E. Dunin-Borkowski, M. E. Scheinfein, J. Moulin, C. Duhamel, F. Mazaleyrat, Y. Champion, Vortex flux channeling in magnetic nanoparticle chains, Phys. Rev. Lett. 91 (25) (2003) 257207.

[15] N. A. Usov, S. Peschany, J. Magn. Magn. Mat. 118 (1993) L290.

[16] W. F. B. Jr., Micromagnetics, John Wiley and Sons, New York, 1963.

[17] J. Moulin, F. Mazaleyrat, Hysteresis cycles of soft nanoparticles, influence of size, shape and material, J. Magn. Magn. Mat. 290-291 (2005) 540-543. 